\title{
New $C$-aryl alditols from Diels-Alder adducts of sugar nitroalkenes
}

\author{
Noelia Araújo, Manuel Baños, María V. Gil, Luis E. Cáceres, Emilio Román, * \\ and José A. Serrano
}

Departamento de Química Orgánica e Inorgánica, Facultad de Ciencias, Universidad de

Extremadura, E-06071 Badajoz, Spain

E-mail: roman@unex.es

\begin{abstract}
2-Nitro-, 2-amino- and 2-acetamido-1-(penta- $O$-acetylpentitol-1'-yl)benzenes were prepared using previously reported Diels-Alder cycloadducts obtained from sugar-derived nitroalkenes with D-galacto and D-manno configurations as starting materials. Deacetylation and oxidative cleavage of the sugar side-chain of nitrobenzene pentitol peracetates yielded nitrobenzaldehydes. From 2-nitro-1-(D-galacto-pentitol-1'-yl)benzene also 1',4'- and 2',5'-anhydro derivatives were synthesized.
\end{abstract}

Keywords: Nitro compounds, $C$-aryl alditols, anhydro derivatives, $C$-nucleosides, aromatization

\section{Introduction}

Compounds with an open-chain monosaccharide unit linked through a $\mathrm{C}-\mathrm{C}$ single bond to a hetero- or carboaromatic ring have received attention due to their diverse biological properties. ${ }^{1-8}$ Thus, the nitrobenzene derivative chloramphenicol ${ }^{1,2}$ inhibits protein synthesis, the methoxybenzene derivative karacilin ${ }^{3}$ possesses antiviral activity in vitro against herpes viruses. This type of substances, namely $C$-aryl alditols, can be considered as acyclo- $C$-nucleoside analogues that by intramolecular dehydration could provide $C$-nucleosides; some of the latter, either natural or synthetic, have been reported to have a broad range of useful antitumor, antifungal and antibiotic properties, thus encouraging the development of methodologies toward this class of products. ${ }^{8-11}$ Besides, the $C$-aryl alditol substructure is also present in some natural products, like the 5-(4aminophenyl)-1,2,3,4-tetrahydroxypentane moiety of methanopterin, a cofactor involved in the biological reduction of $\mathrm{CO}_{2}$ to $\mathrm{CH}_{4}{ }^{12}$

Based on our previous experience with Diels-Alder reactions of sugar derivatives, ${ }^{13-15}$ we report herein the synthesis of several $C$-aryl alditols. Cycloadducts obtained from D-galacto-and D-manno-3,4,5,6,7-penta-O-acetyl-1,2-dideoxy-1-nitrohept-1,2-enitols $\mathbf{1 a},{ }^{16} \mathbf{1 b}^{17}$ (Figure 1) with furan, 1-acetoxy-, or 1-trimethylsilyloxybuta-1,3-dienes were used as starting materials. As an 
example of an acid-catalyzed dehydration of the polyhydroxyalkyl chain in 1-(D-galactopentitol-1'-yl)-2-nitrobenzene 6c, we describe the preparation of 2',5'- and 1',4'-anhydro derivatives 10 and 11.

\section{Results and Discussion}

Upon treatment with $\mathrm{DBU}$ in dichloromethane at room temperature both the D-galacto cycloadduct $\mathbf{2} \mathbf{a}^{15}$ and a 50:50 mixture of $\mathbf{2 a}$ and its stereoisomer $\mathbf{3} \mathbf{a}^{15}$ underwent aromatization by anti- and syn-E2 elimination of acetic acid, thus furnishing 1-(1',2',3',4',5'-penta- $O$-acetyl-Dgalacto-pentitol-1'-yl)-2-nitrobenzene 6a in good yields (Figure 1). Similarly, a 65:35 mixture of D-manno adduct $\mathbf{4} \mathbf{b}^{15}$ and its stereoisomer $\mathbf{5 b}^{15}$ afforded 1-(1',2',3',4',5'-penta- $O$-acetyl-Dmanno-pentitol-1'-yl)-2-nitrobenzene $\mathbf{6 b}$ (85\%). By comparison with closely related reactions, the milder conditions now used for these processes are noticeable: Treatment of $\mathbf{2 a}$ or $\mathbf{4} \mathbf{b}$ with sodium acetate in boiling THF only induced anti-elimination of acetic acid, yielding nitrocyclohexadienes; ${ }^{15}$ under these conditions, stereoisomers of $\mathbf{2 a}$ and $\mathbf{4 b}$ did not undergo the desired syn-elimination, probably because of the unfavorable conformation that has to be adopted by the cyclohexene ring.

Compound $\mathbf{6 b}$ was also obtained by potassium carbonate-induced elimination of acetic acid from the crude mixture of 7 -oxanorbornene stereoisomers $7 \mathbf{b},{ }^{13}$ followed by treatment of the resulting product with acetic anhydride in pyridine at room temperature. As previously reported, ${ }^{18}$ this reaction could involve a base-induced $\beta$-elimination of the heteroatom bridge, followed by aromatization as the result of the elimination of acetic acid. The procedure is very simple and easy, and could be an alternative to other methods that have been used in related reactions, in which 7-oxabicyclo[2.2.1] hept-2-ene systems yielded substituted benzenes by treatment with $\mathrm{TiCl}_{4}-\mathrm{LiAlH}_{4}{ }^{19,20}$

Determination of structures of nitrobenzene pentitol peracetates $6 \mathbf{a}$ and $\mathbf{6 b}$ is based on their analytical and spectroscopic data. The ${ }^{1} \mathrm{H}$ and ${ }^{13} \mathrm{C}$ NMR spectra of both compounds show four protons and six carbon atoms in the aromatic region; the values of the vicinal proton coupling constants in the sugar side-chain are similar to those of their starting materials, ${ }^{15}$ thus supporting the same extended conformation in the acyclic carbohydrate moiety.

Treatment of $\mathbf{6 a}$ and $\mathbf{6 b}$ with potassium carbonate in $90 \%$ methanol yielded the deacetylated derivatives $\mathbf{6 c}$ and $\mathbf{6 d}$, respectively. Oxidative cleavage of the pentahydroxypentyl side-chains in

$\mathbf{6 c}$ and $\mathbf{6 d}$ with sodium metaperiodate in $\mathrm{MeOH} / \mathrm{H}_{2} \mathrm{O}(1: 1)$ led to 2-nitrobenzaldehyde $\mathbf{6 f}$, $^{21}$ thus supporting the proposed structures of their respective starting materials and demonstrating that they differ merely in the configuration of their sugar chains.

Following the method described by Cowan, ${ }^{22}$ reduction of the nitro group in nitrobenzene alditols $6 \mathbf{a}$ and $\mathbf{6 b}$ with $\mathrm{NaBH}_{4} / \mathrm{Cu}(\mathrm{OAc})_{2}$ in methanol yielded the corresponding aniline

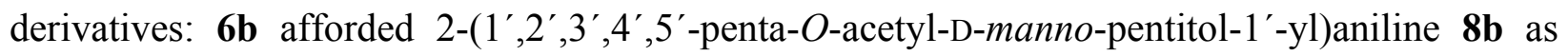
the only product, whereas $6 \mathbf{6}$ led to a $2: 1$ mixture of $2-\left(1^{\prime}, 2^{\prime}, 3^{\prime}, 4^{\prime}, 5^{\prime}\right.$-penta-O-acetyl-D-galacto- 


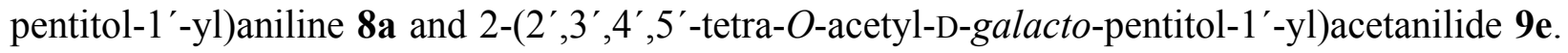
The formation of the latter product is explained by an intramolecular migration of the acetyl group at $\mathrm{C}-1^{\prime}$ to the amino group. The difference in behavior of $\mathbf{6 a}$ and $\mathbf{6 b}$ suggests that the migration could be due to spatial proximity of $\mathrm{C}-1$ ' acetate and the amino group in the D-galacto configuration of the sugar side-chain, which is not the case with the D-manno configuration. Supporting evidence of the structure of acetanilide $9 \mathbf{e}$ is provided by the chemical shift of $\mathrm{H}-1^{\prime}$ at $\delta 5.69$ in $9 \mathbf{e}$, which is shifted downfield to $\delta 6.06$ in the peracetylated derivative $9 \mathbf{a}^{23}$

In order to explore the dehydrating cyclization of $C$-aryl alditols, nitrobenzene pentitol $\mathbf{6 c}$ was refluxed in $1 \%$ sulfuric acid in isopropyl alcohol. Compounds $10(62 \%)$ and $11(20 \%)$ with $2^{\prime}, 5^{\prime}$-anhydro and $1^{\prime}, 4^{\prime}$-anhydro rings, respectively, were isolated. These results agree with those previously reported: ${ }^{24}$ When the sugar chain is linked to a $\pi$-deficient heterocycle, $\mathrm{C}-1^{\prime}$ is usually not involved in the cyclization process, and $2^{\prime}, 5^{\prime}$-anhydro derivatives are formed. Accordingly, the ${ }^{1} \mathrm{H}$ NMR spectrum of $\mathbf{1 1}$ in DMSO- $d_{6}$ displays a triplet signal corresponding to the hydroxyl group at $\mathrm{C}-5^{\prime}$; such a signal is absent in the spectrum of 10. The ${ }^{13} \mathrm{C}$ NMR chemical shifts are similar to those reported for closely related compounds; ${ }^{25}$ the signal for C-5' appears at $\delta 73.1$ for 10, and at $\delta 60.0$ for $\mathbf{1 1}$ in agreement with the type of cyclization proposed.

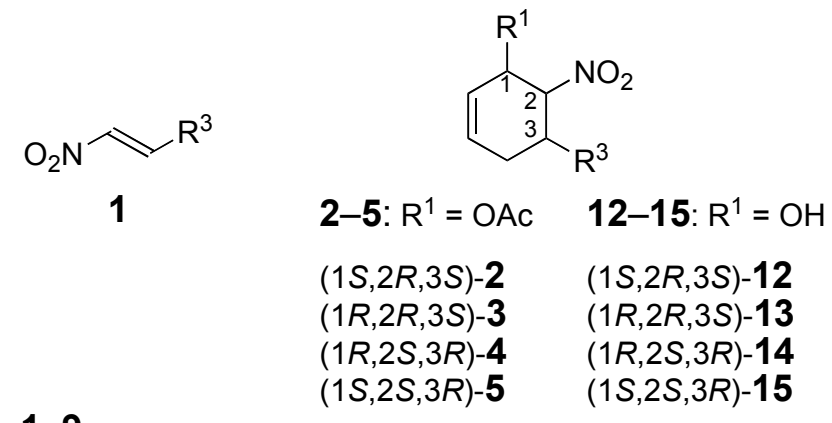

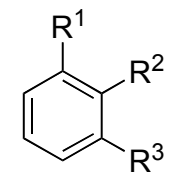

6: $\mathrm{R}^{1}=\mathrm{H}, \mathrm{R}^{2}=\mathrm{NO}_{2}$

8: $\mathrm{R}^{1}=\mathrm{H}, \mathrm{R}^{2}=\mathrm{NH}_{2}$

9: $R^{1}=H, R^{2}=N H A C$

16: $\mathrm{R}^{1}=\mathrm{OH}, \mathrm{R}^{2}=\mathrm{NO}_{2}$

17: $\mathrm{R}^{1}=\mathrm{OAc}, \mathrm{R}^{2}=\mathrm{NO}_{2}$<smiles>[R]C1C2C=CC(C2)C1[N+](=O)[O-]</smiles>

7
$1-9$,

12-17 a:

$\mathrm{R}^{3}=$

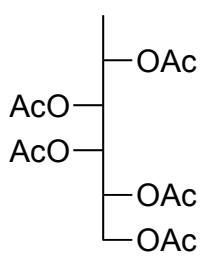

b:

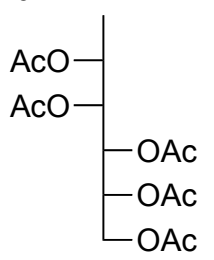

c:

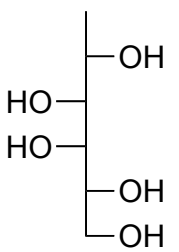

d:

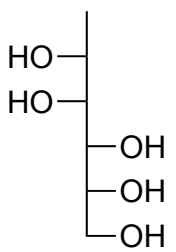

e:

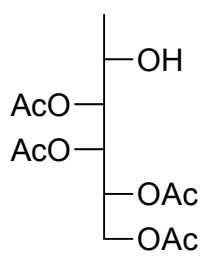

f:

$\mathrm{CH}=\mathrm{O}$

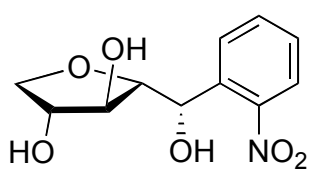

10

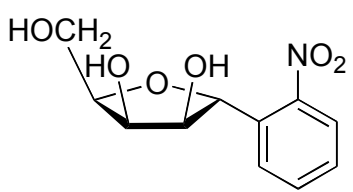

11

Figure 1 
Concerning the dehydration mechanism, we believe that the formation of $\mathbf{1 0}$ probably involves an attack of the C-2' hydroxyl group at C-5' displacing its protonated hydroxyl group. ${ }^{26}$ The magnitude of the coupling constant $\left(J_{2^{\prime}, 3^{\prime}}=3.5 \mathrm{~Hz}\right)$ in $\mathbf{1 0}$ supports the $\alpha$-anomeric configuration; this is within the range of related 3,6-anhydro-D-galactose derivatives: ${ }^{27} \mathrm{~A}$ small coupling constant $\left({ }^{3} J=0.5-4.0 \mathrm{~Hz}\right)$ has been observed between the proton at the ring carbon bearing the $C$-substituent and the proton at the vicinal ring carbon.

Probably, the cyclization leading to $\mathbf{1 1}$ involves a benzylic carbocation intermediate generated in acid medium, and accordingly, proof of its structure cannot be based on mechanistic grounds. The $\alpha$-anomeric configuration is in agreement with the absence of a NOESY effect between the H-1' and H-4', and is consistent with the steric hindrance that would be present in the transition state leading to the $\beta$-anomer with all substituents in the furanoid ring cis oriented; also, the coupling constant $\left(J_{1^{\prime}, 2^{\prime}}=7.8 \mathrm{~Hz}\right)$ is similar to that previously reported $\left({ }^{3} J=8.0 \mathrm{~Hz}\right)$ for 1-benzyl-4,5,6,7-tetrahydro-2- $\alpha$-D-lyxo-furanosyl-6,6-dimethylindol-4-one. ${ }^{28}$

On the other hand, 3-glyco-2-nitrophenols $\mathbf{1 6 a}$ and $\mathbf{1 6 b}$ were prepared from crude hydrolysis mixtures ${ }^{15}$ of the corresponding cycloadducts $\mathbf{1 2 - 1 5}$ by treatment with dimethylsulfoxide and acetic anhydride. As described previously, ${ }^{29}$ these reagents oxidize primary or secondary alcohols to carbonyl compounds, whereas in our case the oxidation led to nitrophenols 16a and $\mathbf{1 6 b}$, probably through the enol form of the initially formed cyclohexenone. The assignment of structures 16a and 16b is based on spectroscopic data as well as on those obtained from the respective hexaacetylated derivatives $17 \mathbf{a}$ and $\mathbf{1 7 b}$, or the deacetylated derivatives $\mathbf{1 6 c}$ and $\mathbf{1 6 d}$. Oxidative cleavage of the pentahydroxypentyl chains of the latter two compounds with sodium metaperiodate in $\mathrm{MeOH} / \mathrm{H}_{2} \mathrm{O}$ (1:1) afforded, in both cases, the known 3-hydroxy-2nitrobenzaldehyde $\mathbf{1 6} \mathbf{f}^{30}$ thus providing additional support for structures $\mathbf{1 6 a}$ and $\mathbf{1 6 b}$.

In conclusion, Diels-Alder cycloadducts obtained from sugar-derived nitroalkenes with furan, 1-acetoxy-, or 1-trimethylsilyloxybuta-1,3-diene are suitable precursors of $C$-aryl alditols. Intramolecular cyclization of the sugar chains leads to $C$-nucleoside analogues. The potential of the nitro group, easily convertible to other functionalities, opens an access to a variety of this class of compounds.

\section{Experimental Section}

General. Solutions were concentrated at reduced pressure below $40{ }^{\circ} \mathrm{C}$. Silica Gel 60 (Merck, 230-400 mesh ASTM) was used for column chromatography, which was carried out using a drycolumn mode. ${ }^{31}$ Thin layer chromatography (TLC) was performed on precoated Merck Kieselgel $60 \mathrm{GF}_{254}$ aluminium-backed plates; visualization with UV light or iodine vapor. Preparative thin layer chromatography (PTLC) was performed using silica gel (Merck $60 \mathrm{GF}_{254}$ ). Reagents were used as supplied by Aldrich Chemical Co. NMR spectra were recorded on a Bruker AC/PC spectrometer $\left(400.13 \mathrm{MHz}\right.$ for ${ }^{1} \mathrm{H}, 100.62 \mathrm{MHz}$ for ${ }^{13} \mathrm{C}$ ) with TMS or residual $\mathrm{CHCl}_{3}$ or DMSO as internal standards. Evaluation of NMR signals is based on spin decoupling, heteronuclear 
chemical shift correlation spectroscopy and DEPT experiments. IR spectra were recorded on Perkin-Elmer 399 and FT-IR MIDAC Corporation spectrophotometers. Solid samples were examined as $\mathrm{KBr}$ disks, and liquids as thin films on $\mathrm{NaCl}$ plates. Melting points were determined in open capillary tubes on an Electrothermal 8100 capillary melting point apparatus. Optical rotations were determined at $20 \pm 2{ }^{\circ} \mathrm{C}$ with a Perkin-Elmer 241 polarimeter.

1-(1',2', 3', $\mathbf{4}^{\prime}, 5^{\prime}$-Penta- $O$-acetyl-D-galacto-pentitol-1'-yl)-2-nitrobenzene (6a). To a stirred solution of $(1 S, 2 R, 3 S)-1-O$-acetyl-3-(1',2',3',4',5'-penta- $O$-acetyl-D-galacto-pentitol-1'-yl)-2nitrocyclohex-5-en-1-ol ${ }^{15}$ (2a; $\left.0.70 \mathrm{~g}, 1.28 \mathrm{mmol}\right)$ in $\mathrm{CH}_{2} \mathrm{Cl}_{2}(6 \mathrm{~mL})$ was added DBU $(0.15 \mathrm{~mL}$, $1.00 \mathrm{mmol}$ ). After $16 \mathrm{~h}$ at room temperature, the mixture was poured onto ice-cold water (50 $\mathrm{mL})$, extracted with $\mathrm{CH}_{2} \mathrm{Cl}_{2}(3 \times 25 \mathrm{~mL})$; the combined extracts were washed successively with $\mathrm{HCl}(1 \mathrm{M}, 2 \times 25 \mathrm{~mL})$, saturated aqueous $\mathrm{NaHCO}_{3}(2 \times 25 \mathrm{~mL})$, and water $(2 \times 25 \mathrm{~mL})$. The organic layer was decolorized with activated charcoal, dried $\left(\mathrm{MgSO}_{4}\right)$, filtered and evaporated to yield $\mathbf{6 a}$ as a pale yellow oil that crystallized from $\mathrm{Et}_{2} \mathrm{O}$ /petroleum ether $(0.51 \mathrm{~g}, 82 \%)$; mp 91$93{ }^{\circ} \mathrm{C} ; R_{f} 0.29\left(\mathrm{Et}_{2} \mathrm{O} /\right.$ petroleum ether, 2:1); $[\alpha]_{\mathrm{D}}+5.6\left(c 0.50, \mathrm{CHCl}_{3}\right)$; IR $(\mathrm{KBr}) \tilde{v}_{\max }\left(\mathrm{cm}^{-1}\right)$ : $3020\left(\mathrm{H}-\mathrm{C}_{\mathrm{ar}}\right), 1735(\mathrm{C}=\mathrm{O}), 1540,1375\left(\mathrm{NO}_{2}\right), 1230,1060(\mathrm{C}-\mathrm{O}-\mathrm{C}) ;{ }^{1} \mathrm{H}$ NMR $\left(\mathrm{CDCl}_{3}\right): \delta 8.12$ $\left(1 \mathrm{H}, \mathrm{dd}, J_{3,5}=1.3 \mathrm{~Hz}, J_{3,4}=8.1 \mathrm{~Hz}, \mathrm{H}-3\right), 7.7-7.4(3 \mathrm{H}, \mathrm{m}, \mathrm{H}-4, \mathrm{H}-5, \mathrm{H}-6), 6.55\left(1 \mathrm{H}, \mathrm{d}, J_{1^{\prime}, 2^{\prime}}=\right.$ $\left.1.2 \mathrm{~Hz}, \mathrm{H}-1^{\prime}\right), 5.69\left(1 \mathrm{H}, \mathrm{dd}, J_{2^{\prime}, 3^{\prime}}=10.1 \mathrm{~Hz}, J_{1^{\prime}, 2^{\prime}}=1.2 \mathrm{~Hz}, \mathrm{H}-2^{\prime}\right), 5.59\left(1 \mathrm{H}, \mathrm{dd}, J_{3^{\prime}, 4^{\prime}}=1.8 \mathrm{~Hz}\right.$, $\left.J_{2^{\prime}, 3^{\prime}}=10.1 \mathrm{~Hz}, \mathrm{H}-3^{\prime}\right), 5.38\left(1 \mathrm{H}, \mathrm{m}, \mathrm{H}-4^{\prime}\right), 4.34\left(1 \mathrm{H}, \mathrm{dd}, J_{4^{\prime}, 5^{\prime}}=4.8 \mathrm{~Hz}, J_{5^{\prime}, 5^{\prime \prime}}=11.5 \mathrm{~Hz}, \mathrm{H}-5^{\prime}\right)$, $3.90\left(1 \mathrm{H}, \mathrm{dd}, J_{4^{\prime}, 5^{\prime \prime}}=7.6 \mathrm{~Hz}, J_{5^{\prime}, 5^{\prime \prime}}=11.5 \mathrm{~Hz}, \mathrm{H}-5^{\prime \prime}\right), 2.20,2.17,2.04,2.01,1.83$ (each 3H, $5 \mathrm{~s}, 5$ $\left.\mathrm{CH}_{3}\right) ;{ }^{13} \mathrm{C} \mathrm{NMR}\left(\mathrm{CDCl}_{3}\right): \delta 170.4,170.3,170.0,169.6,168.8\left(\mathrm{OCOCH}_{3}\right), 147.0(\mathrm{C}-2), 132.9(\mathrm{C}-$ 1), 133.3, 129.2, 127.9, 125.4 (C-3, C-4, C-5, C-6), 68.9, 68.1, 68.0, 67.6 (C-1', C-2', C-3', C$\left.4^{\prime}\right), 62.1\left(\mathrm{C}-5^{\prime}\right), 20.7,20.6,20.5,20.1\left(\mathrm{CH}_{3}\right)$. Anal. Calcd. for $\mathrm{C}_{21} \mathrm{H}_{25} \mathrm{NO}_{12}$ : C, 52.17; H, 5.21; N, 2.90. Found: C, 52.06; H, 5.27; N, 2.76.

Following this procedure, a 50:50 mixture of $\mathbf{2 a}$ and diastereoisomer 3a yielded $\mathbf{6 a}(75 \%)$.

\section{1-(1',2',3',4',5'-Penta- $O$-acetyl-D-manno-pentitol-1'-yl)-2-nitrobenzene (6b).}

Method A: Following the procedure as described above for the preparation of 6a, a 65:35 mixture of $(1 R, 2 S, 3 R)$ - and $(1 S, 2 S, 3 R)-1-O$-acetyl-3-(1',2',3',4',5'-penta- $O$-acetyl-D-mannopentitol-1'-yl)-2-nitrocyclohex-5-en-1-ol ${ }^{15}(\mathbf{4 b}$ and $\mathbf{5 b})$ afforded $\mathbf{6 b}(85 \%)$ as an oil; $R_{f} 0.23$ $\left(\mathrm{Et}_{2} \mathrm{O} /\right.$ petroleum ether, $\left.2: 1\right) ;[\alpha]_{\mathrm{D}}+2.5\left(c 0.40, \mathrm{CHCl}_{3}\right)$; IR (film) $\tilde{v}_{\max }\left(\mathrm{cm}^{-1}\right): 3040\left(\mathrm{H}-\mathrm{C}_{\mathrm{ar}}\right)$, $1750(\mathrm{C}=\mathrm{O}), 1545,1380\left(\mathrm{NO}_{2}\right), 1230,1070(\mathrm{C}-\mathrm{O}-\mathrm{C}) ;{ }^{1} \mathrm{H} \mathrm{NMR}\left(\mathrm{CDCl}_{3}\right): \delta 7.86\left(1 \mathrm{H}, \mathrm{d}, J_{3,4}=\right.$ $7.8 \mathrm{~Hz}, \mathrm{H}-3), 7.61\left(1 \mathrm{H}, \mathrm{d}, J_{5,6}=4.8 \mathrm{~Hz}, \mathrm{H}-6\right), 7.47\left(1 \mathrm{H}, \mathrm{dd}, J_{4,5}=3.4 \mathrm{~Hz}, J_{3,4}=7.8 \mathrm{~Hz}, \mathrm{H}-4\right)$, $7.44\left(1 \mathrm{H}, \mathrm{dd}, J_{4,5}=3.4 \mathrm{~Hz}, J_{5,6}=4.7 \mathrm{~Hz}, \mathrm{H}-5\right), 6.50\left(1 \mathrm{H}, \mathrm{d}, J_{1^{\prime}, 2^{\prime}}=9.9 \mathrm{~Hz}, \mathrm{H}-1^{\prime}\right), 5.61(\mathrm{dd}, 1 \mathrm{H}$, $\left.J_{3^{\prime}, 4^{\prime}}=9.4 \mathrm{~Hz}, J_{2^{\prime}, 3^{\prime}}=1.7 \mathrm{~Hz}, \mathrm{H}-3^{\prime}\right), 5.52\left(1 \mathrm{H}, \mathrm{dd}, J_{2^{\prime}, 3^{\prime}}=1.8 \mathrm{~Hz}, J_{1^{\prime}, 2^{\prime}}=10.0 \mathrm{~Hz}, \mathrm{H}-2^{\prime}\right), 5.08$ $\left(1 \mathrm{H}, \mathrm{ddd}, J_{3^{\prime}, 4^{\prime}}=9.5 \mathrm{~Hz}, J_{4^{\prime}, 5^{\prime}}=2.7 \mathrm{~Hz}, J_{4^{\prime}, 5^{\prime \prime}}=4.8 \mathrm{~Hz}, \mathrm{H}-4^{\prime}\right), 4.24\left(1 \mathrm{H}, \mathrm{dd}, J_{4^{\prime}, 5^{\prime}}=2.7 \mathrm{~Hz}, J_{5^{\prime}, 5^{\prime \prime}}\right.$ $\left.=10.0 \mathrm{~Hz}, \mathrm{H}-5^{\prime}\right), 4.13\left(1 \mathrm{H}, \mathrm{dd}, J_{4^{\prime}, 5^{\prime \prime}}=4.7 \mathrm{~Hz}, J_{5^{\prime}, 5^{\prime \prime}}=10.0 \mathrm{~Hz}, \mathrm{H}-5^{\prime \prime}\right), 2.21,2.11,2.06,2.04$, 1.80 (each $\left.3 \mathrm{H}, 5 \mathrm{~s}, 5 \mathrm{CH}_{3}\right) ;{ }^{13} \mathrm{C} \mathrm{NMR}\left(\mathrm{CDCl}_{3}\right): \delta 170.3,169.7,169.6,169.4,169.3\left(\mathrm{OCOCH}_{3}\right)$, 148.9 (C-2), 132.9, 129.3, 128.3, 124.2 (C-3, C-4, C-5, C-6), 131.1 (C-1), 70.6 (C-1'), 67.6, 67.2, 66.1 (C-2', C-3', C-4'), 61.7 (C-5'), 20.5, 20.4, $20.2\left(\mathrm{CH}_{3}\right)$. 
Method B: To a solution of the crude mixture of oxanorbornenes $7^{13}(0.30 \mathrm{~g}, 0.60 \mathrm{mmol})$ in $\mathrm{MeOH}(90 \%, 8 \mathrm{~mL})$ was added $\mathrm{K}_{2} \mathrm{CO}_{3}(0.35 \mathrm{~g}, 2.53 \mathrm{mmol})$. After stirring for $1 \mathrm{~h}$ at room temperature the resulting solution was neutralized with Amberlite IR-120 $\left(\mathrm{H}^{+}\right)$resin, then filtered and the filtrate concentrated to a residual oil that was treated with pyridine $(1.5 \mathrm{~mL})$ and $\mathrm{Ac}_{2} \mathrm{O}$ $(1.5 \mathrm{~mL})$. After $14 \mathrm{~h}$ at room temperature, the crude product was poured onto ice cold water (50 $\mathrm{mL})$, extracted with $\mathrm{CH}_{2} \mathrm{Cl}_{2}(3 \times 25 \mathrm{~mL})$. The extracts were combined, washed succesively with $\mathrm{HCl}(1 \mathrm{M}, 2 \times 25 \mathrm{~mL})$, saturated aqueous $\mathrm{NaHCO}_{3}(2 \times 25 \mathrm{~mL})$, and water $(2 \times 25 \mathrm{~mL})$. Upon drying $\left(\mathrm{MgSO}_{4}\right)$ and concentration a pure (by TLC) oil $\mathbf{6 b}$ was obtained $(0.27 \mathrm{~g}, 94 \%)$.

2-Nitro-1-(D-galacto-pentitol-1' 'yl)benzene (6c). To a solution of 1-(1',2',3',4',5' -penta- $O$ acetyl-D-galacto-pentitol-1' -yl)-2-nitrobenzene (6a, $1.50 \mathrm{~g}, 3.10 \mathrm{mmol})$ in $\mathrm{MeOH}(90 \%, 38 \mathrm{~mL})$ was added $\mathrm{K}_{2} \mathrm{CO}_{3}(1.70 \mathrm{~g}, 12.30 \mathrm{mmol})$. After stirring for $24 \mathrm{~h}$ at room temperature the reaction mixture was neutralized with Amberlite IR-120 $\left(\mathrm{H}^{+}\right)$and evaporated to yield a colorless oil 6c (0.74 g, 88\%). $R_{f} 0.32$ (benzene/MeOH, 3:1); $[\alpha]_{\mathrm{D}}-120.0$ (c 0.50, pyridine); IR (film) $\tilde{v}_{\max }\left(\mathrm{cm}^{-}\right.$ 1) 3600-3100 (O-H), $3020\left(\mathrm{H}-\mathrm{C}_{\mathrm{ar}}\right), 1550,1360\left(\mathrm{NO}_{2}\right), 1215,1080(\mathrm{C}-\mathrm{O}-\mathrm{C}) ;{ }^{1} \mathrm{H}$ NMR (DMSO$\left.d_{6}\right): \delta 7.90\left(1 \mathrm{H}, \mathrm{d}, J_{3,4}=6.9 \mathrm{~Hz}, \mathrm{H}-3\right), 7.85\left(1 \mathrm{H}, \mathrm{dd}, J_{5,6}=8.0 \mathrm{~Hz}, J_{4,6}=1.0 \mathrm{~Hz}, \mathrm{H}-6\right), 7.68(1 \mathrm{H}, \mathrm{t}$, $\left.J_{3,4}=J_{4,5}=7.2 \mathrm{~Hz}, \mathrm{H}-4\right), 7.46\left(1 \mathrm{H}, \mathrm{t}, J_{4,5}=J_{5,6}=7,6 \mathrm{~Hz}, \mathrm{H}-5\right), 5.48\left(1 \mathrm{H}, \mathrm{s}, \mathrm{H}-1^{\prime}\right), 4.5-3.9(5 \mathrm{H}$, m, $5 \mathrm{OH}, \mathrm{D}_{2} \mathrm{O}$ exchangeable), 3.8-3.4 (5H, m, H-2', H-3' H-4', H-5', H-5 ' '); ${ }^{\prime 3} \mathrm{C}$ NMR (DMSO$\left.d_{6}\right): \delta 147.8$ (C-2), 139.0 (C-1), 132.2, 130.7, 127.6, 123.3 (C-3, C-4, C-5, C-6), 72.5 (C-1'), $70.1,69.8,66.6\left(\mathrm{C}-2^{\prime}, \mathrm{C}-3^{\prime}, \mathrm{C}-4^{\prime}\right), 63.2\left(\mathrm{C}-5^{\prime}\right)$.

2-Nitro-1-(D-manno-pentitol-1' -yl)benzene (6d). Following the procedure as described for the preparation of $\mathbf{6 c}, 1-\left(1^{\prime}, 2^{\prime}, 3^{\prime}, 4^{\prime}, 5^{\prime}\right.$-penta- $O$-acetyl-D-manno-pentitol-1' $\left.-y 1\right)-2$-nitrobenzene $(\mathbf{6 b}$, $0.80 \mathrm{~g}, 1.65 \mathrm{mmol})$ gave a colorless oil $6 \mathbf{d}(0.37 \mathrm{~g}, 82 \%) ; R_{f} 0.34$ (benzene/MeOH, 3:1); $[\alpha]_{\mathrm{D}}$ -2.2 (c 0.50, pyridine); IR (film) $\tilde{v}_{\max }\left(\mathrm{cm}^{-1}\right)$ 3400-3200 (O-H), $3020\left(\mathrm{H}-\mathrm{C}_{\mathrm{ar}}\right), 1560,1400$ $\left(\mathrm{NO}_{2}\right), 1020(\mathrm{C}-\mathrm{O}-\mathrm{C}) ;{ }^{1} \mathrm{H}$ NMR (DMSO-d 6$): \delta 7.83\left(1 \mathrm{H}, \mathrm{d}, J_{3,4}=7.8 \mathrm{~Hz}, \mathrm{H}-3\right), 7.75\left(1 \mathrm{H}, \mathrm{d}, J_{5,6}\right.$ $=8.0 \mathrm{~Hz}, \mathrm{H}-6), 7.65\left(1 \mathrm{H}, \mathrm{t}, J_{3,4}=J_{4,5}=7.6 \mathrm{~Hz}, \mathrm{H}-4\right), 7.44\left(1 \mathrm{H}, \mathrm{t}, J_{4,5}=7.6 \mathrm{~Hz}, \mathrm{H}-5\right), 5.29(1 \mathrm{H}, \mathrm{d}$, $\left.J_{1^{\prime}, 2^{\prime}}=8.9 \mathrm{~Hz}, \mathrm{H}-1^{\prime}\right), 5.0-3.0\left(5 \mathrm{H}, \mathrm{m}, 5 \mathrm{OH}, \mathrm{D}_{2} \mathrm{O}\right.$ exchangeable), 3.8-3.2 (5H, m, H-2', H-3', H$\left.4^{\prime}, \mathrm{H}-5^{\prime}, \mathrm{H}-5^{\prime \prime}\right) ;{ }^{13} \mathrm{C}$ NMR (DMSO-d $\left.d_{6:}\right): \delta 150.0$ (C-2), 139.6 (C-1), 132.6, 128.9, 127.7, 123.4 (C-3, C-4, C-5, C-6), 73.6 (C-1') , 71.4, 69.6, 67.1 (C-2', C-3', C-4'), 64.0 (C-5').

2-Nitrobenzaldehyde (6f). ${ }^{21}$ To a stirred solution of 2-nitro-1-(D-galacto-pentitol-1'-yl)benzene (6c; $0.15 \mathrm{~g}, 0.55 \mathrm{mmol})$ in $\mathrm{MeOH}(50 \%, 12 \mathrm{~mL})$ was added $\mathrm{NaIO}_{4}(0.53 \mathrm{~g}, 2.48 \mathrm{mmol})$. After 15 min at room temperature, the mixture was extracted with $\mathrm{CH}_{2} \mathrm{Cl}_{2}(3 \times 20 \mathrm{~mL})$, the extracts were combined, washed with water $(2 \times 20 \mathrm{~mL})$, dried $\left(\mathrm{MgSO}_{4}\right)$, and evaporated to yield an oil $(0.068$ g, 82\%); IR and ${ }^{1} \mathrm{H}$ NMR data match those reported for 2-nitrobenzaldehyde. ${ }^{21}$

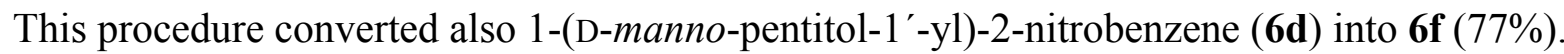

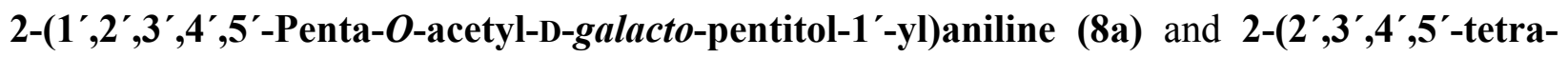
$\boldsymbol{O}$-acetyl-D-galacto-pentitol-1' -yl)acetanilide (9e). To a stirred solution of $1-\left(1^{\prime}, 2^{\prime}, 3^{\prime}, 4^{\prime}, 5^{\prime}\right.$ penta- $O$-acetyl-D-galacto-pentitol-1'-yl)-2-nitrobenzene (6a, $2.00 \mathrm{~g}, 4.00 \mathrm{mmol})$ in $\mathrm{MeOH}(60$ $\mathrm{mL})$ was added a saturated solution of $\mathrm{Cu}(\mathrm{AcO})_{2}(16 \mathrm{~mL})$. The mixture was treated with $\mathrm{NaBH}_{4}$ ( $2.10 \mathrm{~g}$, stepwise; $3 \times 0.70 \mathrm{~g}$, each five $\mathrm{min})$. After $30 \mathrm{~min}$, the reaction mixture was filtered, and the filtrate was diluted with $\mathrm{Et}_{2} \mathrm{O}(120 \mathrm{~mL})$ and washed with saturated aqueous $\mathrm{NaHCO}_{3}(3 \times 50$ 
$\mathrm{mL})$. The aqueous layer was extracted with $\mathrm{Et}_{2} \mathrm{O}(100 \mathrm{~mL})$, the combined organic extracts were dried $\left(\mathrm{MgSO}_{4}\right)$ and evaporated to yield an oil $(1.70 \mathrm{~g}, 91 \%)$, which was shown to be a $2: 1$ mixture of $8 \mathbf{a}$ and $9 \mathbf{e} ; R_{f} 0.32$ and 0.41 , respectively $\left(\mathrm{Et}_{2} \mathrm{O} /\right.$ petroleum ether, 3:1). Although attempts to separate these two products were unsuccessful, their respective NMR data could be obtained from enriched samples (PTLC).

8a: ${ }^{1} \mathrm{H}$ NMR $\left(\mathrm{CDCl}_{3}\right)$ : $\delta 7.66\left(1 \mathrm{H}, \mathrm{d}, J_{5,6}=3.4 \mathrm{~Hz}, \mathrm{H}-6\right), 7.3-7.0(1 \mathrm{H}, \mathrm{m}, \mathrm{H}-3), 7.13(1 \mathrm{H}, \mathrm{br}$, $\left.J_{4,5}=7.2 \mathrm{~Hz}, \mathrm{H}-5\right), 6.69(1 \mathrm{H}, \mathrm{m}, \mathrm{H}-4), 6.28\left(1 \mathrm{H}, \mathrm{d}, J_{1^{\prime}, 2^{\prime}}=5.2 \mathrm{~Hz}, \mathrm{H}-1^{\prime}\right), 5.65\left(1 \mathrm{H}, \mathrm{dd}, J_{1^{\prime}, 2^{\prime}}=5.2\right.$ $\left.\mathrm{Hz}, J_{2^{\prime}, 3^{\prime}}=8.3 \mathrm{~Hz}, \mathrm{H}-2^{\prime}\right), 5.39\left(1 \mathrm{H}, \mathrm{dd}, J_{3^{\prime}, 4^{\prime}}=2.3 \mathrm{~Hz}, J_{2^{\prime}, 3^{\prime}}=8.2 \mathrm{~Hz}, \mathrm{H}-3^{\prime}\right), 5.30\left(1 \mathrm{H}, \mathrm{m}, \mathrm{H}-4^{\prime}\right)$, 4.4-3.8 (2H, m, $2 \mathrm{NH}, \mathrm{D}_{2} \mathrm{O}$ exchangeable), 4.20 (dd, $\left.1 \mathrm{H}, J_{4^{\prime}, 5^{\prime}}=5.1 \mathrm{~Hz}, J_{5^{\prime}, 5^{\prime \prime}}=11.7 \mathrm{~Hz}, \mathrm{H}-5^{\prime}\right)$, $3.85\left(1 \mathrm{H}, \mathrm{dd}, J_{4^{\prime}, 5^{\prime \prime}}=7.1 \mathrm{~Hz}, J_{5^{\prime}, 5^{\prime \prime}}=11.9 \mathrm{~Hz}, \mathrm{H}-5^{\prime \prime}\right), 2.07,2.03,2.01,1.99,1.94$ (each $3 \mathrm{H}, 5 \mathrm{~s}, 5$ $\left.\mathrm{CH}_{3}\right) ;{ }^{13} \mathrm{C}$ NMR $\left(\mathrm{CDCl}_{3}\right): \delta 170.3,170.1,169.9,169.1\left(\mathrm{OCOCH}_{3}\right), 144.4(\mathrm{C}-1), 129.5(\mathrm{C}-3)$, 128.5 (C-5), 119.8 (C-2), 118.5 (C-4), 117.0 (C-6), 70.6 (C-1'), 68.8, 68.6, 67.9 (C-2', C-3', C$\left.4^{\prime}\right), 61.8\left(\mathrm{C}-5^{\prime}\right), 20.6,20.5,20.3\left(\mathrm{CH}_{3}\right)$.

9e: ${ }^{1} \mathrm{H}$ NMR $\left(\mathrm{CDCl}_{3}\right)$ : $\delta 7.96\left(1 \mathrm{H}, \mathrm{d}, J_{5,6}=1.8 \mathrm{~Hz}, \mathrm{H}-6\right), 7.3-7.0(1 \mathrm{H}, \mathrm{m}, \mathrm{H}-3), 7.28(1 \mathrm{H}, \mathrm{s}, \mathrm{NH}$, $\mathrm{D}_{2} \mathrm{O}$ exchangeable), $7.06\left(1 \mathrm{H}\right.$, br d, $\left.J_{4,5}=7.5 \mathrm{~Hz}, \mathrm{H}-5\right), 6.69(1 \mathrm{H}, \mathrm{m}, \mathrm{H}-4), 5.69\left(2 \mathrm{H}, \mathrm{m}, \mathrm{H}-1^{\prime}, \mathrm{H}-\right.$ $\left.2^{\prime}\right), 5.30\left(2 \mathrm{H}, \mathrm{m}, \mathrm{H}-3^{\prime}, \mathrm{H}-4^{\prime}\right), 4.33\left(1 \mathrm{H}, \mathrm{dd}, J_{4^{\prime}, 5^{\prime}}=4.8 \mathrm{~Hz}, J_{5^{\prime}, 5^{\prime \prime}}=11.5 \mathrm{~Hz}, \mathrm{H}-5^{\prime}\right), 3.85(1 \mathrm{H}$, dd, $\left.J_{4^{\prime}, 5^{\prime \prime}}=7.1 \mathrm{~Hz}, J_{5^{\prime}, 5^{\prime \prime}}=11.5 \mathrm{~Hz}, \mathrm{H}-5^{\prime \prime}\right), 3.43\left(1 \mathrm{H}, \mathrm{s}, \mathrm{OH}, \mathrm{D}_{2} \mathrm{O}\right.$ exchangeable), 2.27, 2.13, 1.90, 1.79 (each $\left.3 \mathrm{H}, 4 \mathrm{~s}, 4 \mathrm{CH}_{3}\right) ;{ }^{13} \mathrm{C} \mathrm{NMR}\left(\mathrm{CDCl}_{3}\right): \delta 170.2,169.9,169.8,169.7,168.7\left(\mathrm{OCOCH}_{3}\right)$, $160.8\left(\mathrm{NHCOCH}_{3}\right), 139.3$ (C-1), 135.7 (C-4), 128.2 (C-3), 127.4 (C-5), 119.8 (C-2), 118.5 (C-6), $71.1\left(\mathrm{C}-1^{\prime}\right), 68.2,67.8,67.5$ (C-2', C-3', C-4'), $62.0\left(\mathrm{C}-5^{\prime}\right), 20.8,20.5,20.0\left(\mathrm{OCOCH}_{3}\right.$, $\left.\mathrm{NHCOCH}_{3}\right)$.

2-(1',2',3',4',5'-Penta- $O$-acetyl-D-galacto-pentitol-1'-yl)acetanilide (9a). To a solution of a c.a. 2:1 mixture of 2-(1',2',3',4',5'-penta- $O$-acetyl-D-galacto-pentitol-1'-yl)aniline (8a) and 2$\left(2^{\prime}, 3^{\prime}, 4^{\prime}, 5^{\prime}\right.$-tetra- $O$-acetyl-D-galacto-pentitol-1' -yl)acetanilide $(9 \mathrm{e})(0.40 \mathrm{~g}, 0.88 \mathrm{mmol})$ in pyridine $(4 \mathrm{~mL})$ was added $\mathrm{Ac}_{2} \mathrm{O}(2 \mathrm{~mL})$. After $18 \mathrm{~h}$ at $0{ }^{\circ} \mathrm{C}$ the reaction mixture was poured onto water $(100 \mathrm{~mL})$ and extracted with $\mathrm{CH}_{2} \mathrm{Cl}_{2}(2 \times 50 \mathrm{~mL})$. The combined organic extracts were washed with $\mathrm{HCl}(1 \mathrm{M}, 50 \mathrm{~mL})$ and saturated aqueous $\mathrm{NaHCO}_{3}(50 \mathrm{~mL})$, dried $\left(\mathrm{MgSO}_{4}\right)$, and concentrated to yield 9a as an oil, which crystallized from $\mathrm{Et}_{2} \mathrm{O} /$ petroleum ether $(0.37 \mathrm{~g}$, 86\%); mp 93-95 ${ }^{\circ} \mathrm{C} ; R_{f} 0.14\left(\mathrm{Et}_{2} \mathrm{O} /\right.$ petroleum ether, 2:1); $[\alpha]_{\mathrm{D}}+14.4$ (c 0.50, $\left.\mathrm{CHCl}_{3}\right)$; IR $(\mathrm{KBr}) \tilde{v}_{\max }\left(\mathrm{cm}^{-1}\right) 3420(\mathrm{NH}), 1745(\mathrm{C}=\mathrm{O}), 1365,1225(\mathrm{C}-\mathrm{N}), 1225,1080(\mathrm{C}-\mathrm{O}-\mathrm{C}) ;{ }^{1} \mathrm{H}$ NMR $\left(\mathrm{CDCl}_{3}\right): \delta 8.44\left(1 \mathrm{H}, \mathrm{s}, \mathrm{D}_{2} \mathrm{O}\right.$ exchangeable $\left.\mathrm{NH}\right), 7.74\left(1 \mathrm{H}, \mathrm{d}, J_{5,6}=7.6 \mathrm{~Hz}, \mathrm{H}-6\right), 7.33(2 \mathrm{H}, \mathrm{m}, \mathrm{H}-$ $3, \mathrm{H}-4), 7.18\left(1 \mathrm{H}, \mathrm{t}, J_{4,5}=J_{5,6}=7.6 \mathrm{~Hz}, \mathrm{H}-5\right), 6.06\left(1 \mathrm{H}, \mathrm{d}, J_{1^{\prime}, 2^{\prime}}=5.9 \mathrm{~Hz}, \mathrm{H}-1^{\prime}\right), 5.73\left(1 \mathrm{H}, \mathrm{t}, J_{2^{\prime}, 3^{\prime}}\right.$ $\left.=J_{1^{\prime}, 2^{\prime}}=5.9 \mathrm{~Hz}, \mathrm{H}-2^{\prime}\right), 5.25\left(2 \mathrm{H}, \mathrm{m}, \mathrm{H}-3^{\prime}, \mathrm{H}-4^{\prime}\right), 4.10\left(1 \mathrm{H}, \mathrm{dd}, J_{4^{\prime}, 5^{\prime}}=5.3 \mathrm{~Hz}, J_{5^{\prime}, 5^{\prime \prime}}=11.3 \mathrm{~Hz}\right.$, H-5'), $3.82\left(1 \mathrm{H}, \mathrm{dd}, J_{4^{\prime}, 5^{\prime \prime}}=7.0 \mathrm{~Hz}, J_{5^{\prime}, 5^{\prime \prime}}=11.3 \mathrm{~Hz}, \mathrm{H}-5^{\prime \prime}\right), 2.24,2.06,1.99,1.96,1.95,1.94$ (each $\left.3 \mathrm{H}, 6 \mathrm{~s}, \mathrm{NHCOCH}_{3}, 5 \mathrm{OCOCH}_{3}\right) ;{ }^{13} \mathrm{C} \mathrm{NMR}\left(\mathrm{CDCl}_{3}\right): \delta 170.1,170.0,169.8,169.5,168.9$, $168.5\left(5 \mathrm{OCOCH}_{3}, \mathrm{NHCOCH}_{3}\right), 135.4$ (C-1), 129.3, 127.9, 125.2 (C-3, C-4, C-5, C-6), 127.0 (C2), 70.2, 69.3, 68.2, 67.2 (C-1', C-2', C-3', C-4'), 61.4 (C-5'), 23.7, 20.4, 20.2, 20.1, 19.9 $\left(\mathrm{NHCOCH}_{3}, 5 \mathrm{OCOCH}_{3}\right)$. Anal. Calcd for $\mathrm{C}_{23} \mathrm{H}_{29} \mathrm{NO}_{11}: \mathrm{C}, 55.75 ; \mathrm{H}, 5.90 ; \mathrm{N}, 2.83$. Found: C, 55.97; H, 6.06; N, 2.78. 
2-(1',2', $3^{\prime}, 4^{\prime}, 5^{\prime}$-Penta-O-acetyl-D-manno-pentitol-1'-yl)aniline (8b). By the same procedure as described for the preparation of $\mathbf{8 a}$ and $\mathbf{9 e}, 1-\left(1^{\prime}, 2^{\prime}, 3^{\prime}, 4^{\prime}, 5^{\prime}\right.$-penta- $O$-acetyl-D-manno-pentitol-1' yl)-2-nitrobenzene $(\mathbf{6 b}, 0.40 \mathrm{~g}, 0.83 \mathrm{mmol})$ yielded $\mathbf{8 b}$ as an oil, which crystallized from $\mathrm{Et}_{2} \mathrm{O} /$ petroleum ether $(0.25 \mathrm{~g}, 67 \%) ; \mathrm{mp} 150-152{ }^{\circ} \mathrm{C} ; R_{f} 0.15\left(\mathrm{Et}_{2} \mathrm{O} /\right.$ petroleum ether, $\left.2: 1\right) ;[\alpha]_{\mathrm{D}}$ $+67.3\left(c 0.60, \mathrm{CHCl}_{3}\right)$; IR (KBr) $\tilde{v}_{\max }\left(\mathrm{cm}^{-1}\right)$ 3490, $3440\left(\mathrm{NH}_{2}\right), 3020\left(\mathrm{H}-\mathrm{C}_{\mathrm{ar}}\right), 1750(\mathrm{C}=\mathrm{O})$, 1375, $1240(\mathrm{C}-\mathrm{N}), 1240,1040(\mathrm{C}-\mathrm{O}-\mathrm{C}) ;{ }^{1} \mathrm{H}$ NMR $\left(\mathrm{CDCl}_{3}\right): \delta 7.16\left(1 \mathrm{H}, \mathrm{d}, J_{3,4}=7.5 \mathrm{~Hz}, \mathrm{H}-3\right)$, $7.08\left(1 \mathrm{H}, \mathrm{t}, J_{4,5}=J_{5,6}=7.5 \mathrm{~Hz}, \mathrm{H}-5\right), 6.71\left(1 \mathrm{H}, \mathrm{t}, J_{3,4}=J_{4,5}=7.5 \mathrm{~Hz}, \mathrm{H}-4\right), 6.62\left(1 \mathrm{H}, \mathrm{d}, J_{5,6}=7.5\right.$ $\mathrm{Hz}, \mathrm{H}-6), 5.81\left(2 \mathrm{H}, \mathrm{m}, \mathrm{H}-1^{\prime}, \mathrm{H}-2^{\prime}\right), 5.62\left(1 \mathrm{H}, \mathrm{dd}, J_{2^{\prime}, 3^{\prime}}=1.1 \mathrm{~Hz}, J_{3^{\prime}, 4^{\prime}}=9.1 \mathrm{~Hz}, \mathrm{H}-3^{\prime}\right), 5.10(1 \mathrm{H}$, m, H-4'), $4.22\left(1 \mathrm{H}, \mathrm{dd}, J_{4^{\prime}, 5^{\prime}}=2.5 \mathrm{~Hz}, J_{5^{\prime}, 5^{\prime \prime}}=12.4 \mathrm{~Hz}, \mathrm{H}-5^{\prime}\right), 4.16\left(2 \mathrm{H}, \mathrm{m}, 2 \mathrm{NH}, \mathrm{D}_{2} \mathrm{O}\right.$ exchangeable), $4.09\left(1 \mathrm{H}, \mathrm{dd}, J_{4^{\prime}, 5^{\prime \prime}}=5.0 \mathrm{~Hz}, J_{5^{\prime}, 5^{\prime \prime}}=12.5 \mathrm{~Hz}, \mathrm{H}-5^{\prime \prime}\right), 2.15,2.07,2.05,2.04,1.79$ (each $\left.3 \mathrm{H}, 5 \mathrm{~s}, 5 \mathrm{CH}_{3}\right) ;{ }^{13} \mathrm{C} \mathrm{NMR}\left(\mathrm{CDCl}_{3}\right): \delta 170.4,169.7,169.5,169.0\left(\mathrm{OCOCH}_{3}\right), 145.2(\mathrm{C}-1)$, 129.5, 128.8 (C-3, C-5), 119.5 (C-2), 118.1, 116.6 (C-4, C-6), 69.1, 68.8, 67.9, 67.3 (C-1', C-2', C-3', C-4'), 61.7 (C-5'), 20.6, 20.4, $19.9\left(\mathrm{CH}_{3}\right)$. Anal. Calcd for $\mathrm{C}_{21} \mathrm{H}_{27} \mathrm{NO}_{10}$ : C, 55.62; H, 6.00; N, 3.09. Found: C, 55.87; H, 5.93; N, 3.06.

$(2 R, 3 R, 4 S)-2-[(S)$-hydroxy[2-nitrophenyl]methyl]tetrahydrofuran-3,4-diol $\quad(10) \quad$ and (2R,3S,4R,5R)-2-(hydroxymethyl)-5-(2-nitrophenyl)tetrahydrofuran-3,4-diol (11). To a solution of 2-nitro-1-(D-galacto-pentitol-1'-yl)benzene (6c; $0.10 \mathrm{~g}, 0.37 \mathrm{mmol})$ in $i$-PrOH $(10$ $\mathrm{mL})$ was added $\mathrm{H}_{2} \mathrm{SO}_{4}(1 \%, 100 \mathrm{~mL})$, and the mixture was refluxed for 4 days. The reaction mixture was neutralized with saturated aqueous $\mathrm{NaHCO}_{3}$, the solvent was evaporated, and the crude residue was subjected to column chromatography (EtOAc/EtOH, 6:1). Concentration of fractions with $R_{f} 0.71$ and 0.64 afforded oils, which were characterized as $\mathbf{1 0}(0.058 \mathrm{~g}, 62 \%)$ and $11(0.019 \mathrm{~g}, 20 \%)$, respectively.

10: $[\alpha]_{\mathrm{D}}+29.1$ (c 0.51, DMSO); IR (film) $\tilde{v}_{\max }\left(\mathrm{cm}^{-1}\right)$ 3500-3100 (O-H), $3030\left(\mathrm{H}-\mathrm{C}_{\mathrm{ar}}\right), 1540$, $1370\left(\mathrm{NO}_{2}\right), 1215,1095(\mathrm{C}-\mathrm{O}) ;{ }^{1} \mathrm{H}$ NMR (DMSO-d $): \delta 7.85\left(1 \mathrm{H}, \mathrm{d}, J_{3,4}=8.0 \mathrm{~Hz}, \mathrm{H}-3\right), 7.81$ $\left(1 \mathrm{H}, \mathrm{d}, J_{5,6}=7.8 \mathrm{~Hz}, \mathrm{H}-6\right), 7.69\left(1 \mathrm{H}, \mathrm{t}, J_{4,5}=J_{5,6}=7.5 \mathrm{~Hz}, \mathrm{H}-5\right), 7.50\left(1 \mathrm{H}, \mathrm{t}, J_{4,5}=J_{3,4}=7.8 \mathrm{~Hz}\right.$, $\mathrm{H}-4), 5.83\left(1 \mathrm{H}, \mathrm{d}, J=5.1 \mathrm{~Hz}, \mathrm{OH}, \mathrm{D}_{2} \mathrm{O}\right.$ exchangeable), $5.23\left(2 \mathrm{H}\right.$, br s, $2 \mathrm{OH}, \mathrm{D}_{2} \mathrm{O}$ exchangeable), $5.19\left(1 \mathrm{H}, \mathrm{d}, J_{1^{\prime}, 2^{\prime}}=3.4 \mathrm{~Hz}, \mathrm{H}-1^{\prime}\right), 3.91\left(2 \mathrm{H}, \mathrm{br} \mathrm{s}, \mathrm{H}-3^{\prime}, \mathrm{H}-4^{\prime}\right), 3.81\left(1 \mathrm{H}, \mathrm{t}, J_{2^{\prime}, 3^{\prime}}=\right.$ $\left.3.5 \mathrm{~Hz}, \mathrm{H}-2^{\prime}\right), 3.77\left(1 \mathrm{H}, \mathrm{dd}, J_{5^{\prime}, 5^{\prime \prime}}=9.1 \mathrm{~Hz}, J_{4^{\prime}, 5^{\prime}}=4.2 \mathrm{~Hz}, \mathrm{H}-5^{\prime}\right), 3.60\left(1 \mathrm{H}, \mathrm{dd}, J_{4^{\prime}, 5^{\prime \prime}}=2.7 \mathrm{~Hz}\right.$, $\mathrm{H}-5^{\prime \prime}$ ); ${ }^{13} \mathrm{C}$ NMR (DMSO-d 6 ): $\delta 148.2$ (C-2), 136.9 (C-1), 132.5, 130.2, 128.2, 123.6 (C-3, C-4, C-5, C-6), 87.2 (C-2'), 77.9, 76.6 (C-3', C-4'), 73.1 (C-5'), 67.4 (C-1'). Anal. Calcd. for $\mathrm{C}_{11} \mathrm{H}_{13} \mathrm{NO}_{6}$ : C, 51.77; H, 5.13; N, 5.49. Found: C, 51.69; H, 5.08; N, 5.45.

11: $[\alpha]_{\mathrm{D}}-34.5$ (c 0.55, DMSO); IR (film) $\tilde{v}_{\max }\left(\mathrm{cm}^{-1}\right): 3500-3100(\mathrm{O}-\mathrm{H}), 3030\left(\mathrm{H}-\mathrm{C}_{\mathrm{ar}}\right), 1545$, $1375\left(\mathrm{NO}_{2}\right), 1220,1150,1080(\mathrm{C}-\mathrm{O}) ;{ }^{1} \mathrm{H} \mathrm{NMR}\left(\mathrm{DMSO}-d_{6}\right): \delta 7.78\left(1 \mathrm{H}, \mathrm{d}, J_{3,4}=7.8 \mathrm{~Hz}, \mathrm{H}-3\right)$, $7.66(2 \mathrm{H}, \mathrm{m}, \mathrm{H}-5, \mathrm{H}-6), 7.51\left(1 \mathrm{H}, \mathrm{t}, J_{4,5}=J_{3,4}=7.6 \mathrm{~Hz}, \mathrm{H}-4\right), 5.17\left(1 \mathrm{H}, \mathrm{d}, J=7.1 \mathrm{~Hz}, 2^{\prime}-\mathrm{OH}\right.$, $\mathrm{D}_{2} \mathrm{O}$ exchangeable), $5.06\left(1 \mathrm{H}, \mathrm{d}, J_{1^{\prime}, 2^{\prime}}=7.8 \mathrm{~Hz}, \mathrm{H}-1^{\prime}\right), 4.99\left(1 \mathrm{H}, \mathrm{d}, J=3.4 \mathrm{~Hz}, 3^{\prime}-\mathrm{OH}, \mathrm{D}_{2} \mathrm{O}\right.$ exchangeable), 4.62 (1H, t, $J=4.9 \mathrm{~Hz}, 5^{\prime}-\mathrm{OH}, \mathrm{D}_{2} \mathrm{O}$ exchangeable), $4.11\left(1 \mathrm{H}, \mathrm{m}, \mathrm{H}-4^{\prime}\right), 4.05$ $\left(1 \mathrm{H}, \mathrm{t}, J_{2^{\prime}, 3^{\prime}}=J_{3^{\prime}, 4^{\prime}}=4.0 \mathrm{~Hz}, \mathrm{H}-3^{\prime}\right), 4.00\left(1 \mathrm{H}, \mathrm{dd}, J_{1^{\prime}, 2^{\prime}}=7.8 \mathrm{~Hz}, J_{2^{\prime}, 3^{\prime}}=4.0 \mathrm{~Hz}, \mathrm{H}-2^{\prime}\right), 3.65(1 \mathrm{H}$, $\left.\mathrm{dd}, J_{5^{\prime}, 5^{\prime \prime}}=10.7 \mathrm{~Hz}, J_{4^{\prime}, 5^{\prime}}=5.1 \mathrm{~Hz}, \mathrm{H}-5^{\prime}\right), 3.50\left(1 \mathrm{H}, \mathrm{dd}, J_{4^{\prime}, 5^{\prime \prime}}=5.6 \mathrm{~Hz}, J_{5^{\prime}, 5^{\prime \prime}}=10.8 \mathrm{~Hz}, \mathrm{H}-5^{\prime \prime}\right)$; ${ }^{13} \mathrm{C}$ NMR (DMSO- $d_{6}$ ): $\delta 149.0$ (C-2), 139.2 (C-1), 132.4, 128.5, 128.3, 123.6 (C-3, C-4, C-5, C- 
6), 82.1, 78.8, 78.2, 71.2 (C-1', C-2', C-3', C-4'), 60 (C-5'). Anal. Calcd. for $\mathrm{C}_{11} \mathrm{H}_{13} \mathrm{NO}_{6}$ : C, 51.77; H, 5.13; N, 5.49. Found: C, 51.60; H, 5.22; N, 5.35.

3-(1',2',3',4',5'-Penta-O-acetyl-D-galacto-pentitol-1'-yl)-2-nitrophenol (16a). A mixture of $(1 S, 2 R, 3 S)$ - and $(1 R, 2 R, 3 S)-3-\left(1^{\prime}, 2^{\prime}, 3^{\prime}, 4^{\prime}, 5^{\prime}\right.$-penta-O-acetyl-D-galacto-pentitol-1' -yl)-2-nitrocyclohex-5-en-1-ol ${ }^{15}$ (12a and 13a, each $1.00 \mathrm{~g}, 1.99 \mathrm{mmol}$ ) was dissolved in DMSO (12.0 mL) and $\mathrm{Ac}_{2} \mathrm{O}(7.8 \mathrm{~mL})$. After $24 \mathrm{~h}$, the crude mixture was concentrated affording 16a as an oil, which was purified by column chromatography $\left(\mathrm{Et}_{2} \mathrm{O} /\right.$ petroleum ether, $\left.1: 1\right)(0.81 \mathrm{~g}, 82 \%) ; R_{f}$ $0.21\left(\mathrm{Et}_{2} \mathrm{O} /\right.$ petroleum ether, $\left.2: 1\right) ;[\alpha]_{\mathrm{D}}+20.0\left(c 0.45, \mathrm{CHCl}_{3}\right)$; IR (film) $\tilde{v}_{\max }\left(\mathrm{cm}^{-1}\right): 3340(\mathrm{OH})$, $3025\left(\mathrm{H}-\mathrm{C}_{\mathrm{ar}}\right), 1750(\mathrm{C}=\mathrm{O}), 1217,1045(\mathrm{C}-\mathrm{O}-\mathrm{C}) ;{ }^{1} \mathrm{HNMR}\left(\mathrm{CDCl}_{3}\right): \delta 9.85(1 \mathrm{H}$, br s, phenolic $\mathrm{OH}, \mathrm{D}_{2} \mathrm{O}$ exchangeable), $7.51\left(1 \mathrm{H}, \mathrm{dd}, J_{4,6}=1.1 \mathrm{~Hz}, J_{5,6}=7.6 \mathrm{~Hz}, \mathrm{H}-6\right), 7.33\left(1 \mathrm{H}, \mathrm{t}, J_{5,6}=J_{4,5}=\right.$ $7.6 \mathrm{~Hz}, \mathrm{H}-5), 7.25\left(1 \mathrm{H}, \mathrm{dd}, J_{4,5}=7.6 \mathrm{~Hz}, J_{4,6}=1.1 \mathrm{~Hz}, \mathrm{H}-4\right), 6.60\left(1 \mathrm{H}, \mathrm{d}, J_{1^{\prime}, 2^{\prime}}=2.0 \mathrm{~Hz}, \mathrm{H}-1^{\prime}\right)$, $5.69\left(1 \mathrm{H}, \mathrm{dd}, J_{2^{\prime}, 3^{\prime}}=10.0 \mathrm{~Hz}, J_{1^{\prime}, 2^{\prime}}=2.1 \mathrm{~Hz}, \mathrm{H}-2^{\prime}\right), 5.62\left(1 \mathrm{H}, \mathrm{dd}, J_{3^{\prime}, 4^{\prime}}=1.6 \mathrm{~Hz}, J_{2^{\prime}, 3^{\prime}}=9.9 \mathrm{~Hz}\right.$, H-3'), $5.34\left(1 \mathrm{H}, \mathrm{m}, \mathrm{H}-4^{\prime}\right), 4.33\left(1 \mathrm{H}, \mathrm{dd}, J_{4^{\prime}, 5^{\prime}}=5.0 \mathrm{~Hz}, J_{5^{\prime}, 5^{\prime \prime}}=11.5 \mathrm{~Hz}, \mathrm{H}-5^{\prime}\right), 3.88(\mathrm{dd}, 1 \mathrm{H}$, $\left.J_{4^{\prime}, 5^{\prime \prime}}=4.0 \mathrm{~Hz}, J_{5^{\prime}, 5^{\prime \prime}}=11.5 \mathrm{~Hz}, \mathrm{H}-5^{\prime \prime}\right), 2.20,2.18,2.02,1.99,1.79\left(\right.$ each $\left.3 \mathrm{H}, 5 \mathrm{~s}, 5 \mathrm{CH}_{3}\right) ;{ }^{13} \mathrm{C}$ NMR $\left(\mathrm{CDCl}_{3}\right): \delta 170.4-168.7\left(\mathrm{OCOCH}_{3}\right), 152.6(\mathrm{C}-1), 149.6(\mathrm{C}-2), 128.6(\mathrm{C}-3), 125.1(\mathrm{C}-5)$, 121.4 (C-4), 110.8 (C-6), 69.5, 68.5, 68.1, 67.7 (C-1', C-2', C-3', C-4'), 62.0 (C-5'), 20.7, 20.5, 20.2, $20.1\left(\mathrm{CH}_{3}\right)$.

1-Acetoxy-3-(1',2',3', $4^{\prime}, 5^{\prime}$-penta- $O$-acetyl-D-galacto-pentitol-1' -yl)-2-nitrobenzene $\quad(17 \mathrm{a})$. Acetylation of $16 \mathbf{a}(0.10 \mathrm{~g}, 0.20 \mathrm{mmol})$ with pyridine $(1.0 \mathrm{~mL})$ and acetic anhydride $(0.5 \mathrm{~mL})$ quantitatively gave hexaacetate 17a as an oil; $R_{f} 0.54\left(\mathrm{Et}_{2} \mathrm{O} /\right.$ petroleum ether, $\left.2: 1\right) ;[\alpha]_{\mathrm{D}}+28.5(c$ 0.60, $\left.\mathrm{CHCl}_{3}\right)$; IR (film) $\tilde{v}_{\max }\left(\mathrm{cm}^{-1}\right): 3040\left(\mathrm{H}-\mathrm{C}_{\mathrm{ar}}\right), 1745(\mathrm{C}=\mathrm{O}), 1225,1050(\mathrm{C}-\mathrm{O}-\mathrm{C}) ;{ }^{1} \mathrm{H}$ NMR $\left(\mathrm{CDCl}_{3}\right): \delta 7.85\left(1 \mathrm{H}, \mathrm{dd}, J_{4,6}=1.2 \mathrm{~Hz}, J_{5,6}=7.7 \mathrm{~Hz}, \mathrm{H}-6\right), 7.47\left(1 \mathrm{H}, \mathrm{t}, J_{5,6}=J_{4,5}=7.7 \mathrm{~Hz}, \mathrm{H}-5\right)$, $7.49\left(1 \mathrm{H}, \mathrm{dd}, J_{4,6}=1.2 \mathrm{~Hz}, J_{4,5}=7.6 \mathrm{~Hz}, \mathrm{H}-4\right), 6.62\left(1 \mathrm{H}, \mathrm{d}, J_{1^{\prime}, 2^{\prime}}=1.9 \mathrm{~Hz}, \mathrm{H}-1^{\prime}\right), 5.71(1 \mathrm{H}, \mathrm{dd}$, $\left.J_{2^{\prime}, 3^{\prime}}=9.8 \mathrm{~Hz}, J_{1^{\prime}, 2^{\prime}}=2.0 \mathrm{~Hz}, \mathrm{H}-2^{\prime}\right), 5.63\left(1 \mathrm{H}, \mathrm{dd}, J_{3^{\prime}, 4^{\prime}}=1.8 \mathrm{~Hz}, J_{2^{\prime}, 3^{\prime}}=9.8 \mathrm{~Hz}, \mathrm{H}-3^{\prime}\right), 5.36(1 \mathrm{H}$, $\left.\mathrm{m}, \mathrm{H}-4^{\prime}\right), 4.38\left(1 \mathrm{H}, \mathrm{dd}, J_{4^{\prime}, 5^{\prime}}=4.8 \mathrm{~Hz}, J_{5^{\prime}, 5^{\prime \prime}}=12.0 \mathrm{~Hz}, \mathrm{H}-5^{\prime}\right), 3.91\left(1 \mathrm{H}, \mathrm{dd}, J_{4^{\prime}, 5^{\prime \prime}}=4.2 \mathrm{~Hz}, J_{5^{\prime}, 5^{\prime \prime}}\right.$ $\left.=11.8 \mathrm{~Hz}, \mathrm{H}-5^{\prime \prime}\right), 2.27,2.20,2.18,2.02,1.99,1.79\left(\right.$ each $\left.3 \mathrm{H}, 6 \mathrm{~s}, 6 \mathrm{CH}_{3}\right) ;{ }^{13} \mathrm{C} \mathrm{NMR}\left(\mathrm{CDCl}_{3}\right): \delta$ 170.2-168.0 $\left(\mathrm{OCOCH}_{3}\right), 154.6$ (C-2), 135.8 (C-1), 130.2 (C-4), 127.7 (C-3), 124.1 (C-5), 116.5 (C-6), 69.1, 68.4, 68.1, 67.6 (C-1', C-2', C-3', C-4'), 62.0 (C-5'), 20.6, 20.5, 20.2, $19.9\left(\mathrm{CH}_{3}\right)$.

3-(1',2',3',4',5'-Penta-O-acetyl-D-manno-pentitol-1'-yl)-2-nitrophenol (16b). Following the procedure described above for the preparation of 16a, a mixture of $(1 S, 2 R, 3 S)-,(1 R, 2 R, 3 S)$-, $(1 R, 2 S, 3 R)-$, and $(1 S, 2 S, 3 R)-3-\left(1^{\prime}, 2^{\prime}, 3^{\prime}, 4^{\prime}, 5^{\prime}\right.$-penta-O-acetyl-D-manno-pentitol-1' -yl)-2-nitrocyclohex-5-en-1-ol ${ }^{15}(\mathbf{1 2 b}-\mathbf{1 5 b})$ afforded $\mathbf{1 6 b}$ as an oil, which was purified by column chromatography $\left(\mathrm{Et}_{2} \mathrm{O}\right.$ /petroleum ether, 1:1) (68\%); $R_{f} 0.26\left(\mathrm{Et}_{2} \mathrm{O} /\right.$ petroleum ether, 2:1); $[\alpha]_{\mathrm{D}}$ -18.0 (c 0.50, $\left.\mathrm{CHCl}_{3}\right)$; IR (film) $\tilde{v}_{\max }\left(\mathrm{cm}^{-1}\right): 3370(\mathrm{OH}), 3030\left(\mathrm{H}-\mathrm{C}_{\mathrm{ar}}\right), 1750(\mathrm{C}=\mathrm{O}), 1215,1065$ $(\mathrm{C}-\mathrm{O}-\mathrm{C}) ;{ }^{1} \mathrm{HNMR}\left(\mathrm{CDCl}_{3}\right): \delta 9.93\left(1 \mathrm{H}\right.$, br s, phenolic $\mathrm{OH}, \mathrm{D}_{2} \mathrm{O}$ exchangeable $), 7.47\left(1 \mathrm{H}, \mathrm{t}, J_{5,6}\right.$ $\left.=J_{4,5}=7.9 \mathrm{~Hz}, \mathrm{H}-5\right), 7.19\left(1 \mathrm{H}, \mathrm{t}, J_{4,6}=1.0 \mathrm{~Hz}, \mathrm{H}-6\right), 7.01\left(1 \mathrm{H}, \mathrm{dd}, J_{4,6}=1.0 \mathrm{~Hz}, J_{4,5}=8.0 \mathrm{~Hz}\right.$, $\mathrm{H}-4), 6.54\left(1 \mathrm{H}, \mathrm{d}, J_{1^{\prime}, 2^{\prime}}=9.6 \mathrm{~Hz}, \mathrm{H}-1^{\prime}\right), 5.64\left(1 \mathrm{H}, \mathrm{dd}, J_{3^{\prime}, 4^{\prime}}=9.0 \mathrm{~Hz}, J_{2^{\prime}, 3^{\prime}}=1.7 \mathrm{~Hz}, \mathrm{H}-3^{\prime}\right), 5.51$ $\left(1 \mathrm{H}, \mathrm{dd}, J_{2^{\prime}, 3^{\prime}}=1.7 \mathrm{~Hz}, J_{1^{\prime}, 2^{\prime}}=9.6 \mathrm{~Hz}, \mathrm{H}-2^{\prime}\right), 5.06\left(1 \mathrm{H}, \mathrm{m}, \mathrm{H}-4^{\prime}\right), 4.22\left(1 \mathrm{H}, \mathrm{dd}, J_{4^{\prime}, 5^{\prime}}=2.8 \mathrm{~Hz}\right.$, $\left.J_{5^{\prime}, 5^{\prime \prime}}=10.5 \mathrm{~Hz}, \mathrm{H}-5^{\prime}\right), 4.10\left(1 \mathrm{H}, \mathrm{dd}, J_{4^{\prime}, 5^{\prime \prime}}=4.7 \mathrm{~Hz}, J_{5^{\prime}, 5^{\prime \prime}}=10.5 \mathrm{~Hz}, \mathrm{H}-5^{\prime \prime}\right), 2.19,2.10,2.06$, 2.02, 1.82, (each 3H, $\left.5 \mathrm{~s}, 5 \mathrm{CH}_{3}\right) ;{ }^{13} \mathrm{C} \mathrm{NMR}\left(\mathrm{CDCl}_{3}\right)$ : $\delta 170.5,169.8,169.6,169.4,169.3$ 
$\left(\mathrm{OCOCH}_{3}\right), 152.9$ (C-1), 147.4 (C-2), 128.6 (C-3), 128.4 (C-5), 121.2 (C-4), 112.8 (C-6), 70.3 $\left(\mathrm{C}-1^{\prime}\right), 68.2,67.5,66.9$ (C-2', C-3', C-4'), 61.8 (C-5'), 21.0, 20.5, 20.3, $20.1\left(\mathrm{CH}_{3}\right)$.

\section{1-Acetoxy-3-(1',2',3',4',5'-penta- $O$-acetyl-D-manno-pentitol-1' 'yl)-2-nitrobenzene}

(17b).

Acetylation of $\mathbf{1 6 b}(0.10 \mathrm{~g}, 0.20 \mathrm{mmol})$ with pyridine $(1.0 \mathrm{~mL})$ and acetic anhydride $(0.5 \mathrm{~mL})$ quantitatively gave hexaacetate $\mathbf{1 7 b}$ as an oil; $R_{f} 0.57\left(\mathrm{Et}_{2} \mathrm{O} /\right.$ petroleum ether, $\left.2: 1\right) ;[\alpha]_{\mathrm{D}}-26.0(c$ 0.50, $\left.\mathrm{CHCl}_{3}\right)$; IR (film) $\tilde{v}_{\max }\left(\mathrm{cm}^{-1}\right): 3030\left(\mathrm{H}-\mathrm{C}_{\mathrm{ar}}\right), 1750(\mathrm{C}=\mathrm{O}), 1225,1045(\mathrm{C}-\mathrm{O}-\mathrm{C})$; ${ }^{1} \mathrm{HNMR}$ $\left(\mathrm{CDCl}_{3}\right): \delta 7.52\left(1 \mathrm{H}, \mathrm{dd}, J_{4,6}=1.2 \mathrm{~Hz}, J_{5,6}=7.7 \mathrm{~Hz}, \mathrm{H}-6\right), 7.58\left(1 \mathrm{H}, \mathrm{t}, J_{5,6}=J_{4,5}=7.6 \mathrm{~Hz}, \mathrm{H}-5\right)$, $7.26\left(1 \mathrm{H}, \mathrm{dd}, J_{4,6}=1.2 \mathrm{~Hz}, J_{4,5}=7.6 \mathrm{~Hz}, \mathrm{H}-4\right), 6.55\left(1 \mathrm{H}, \mathrm{d}, J_{1^{\prime}, 2^{\prime}}=9.2 \mathrm{~Hz}, \mathrm{H}-1^{\prime}\right), 5.73(1 \mathrm{H}, \mathrm{dd}$, $\left.J_{2^{\prime}, 3^{\prime}}=1.6 \mathrm{~Hz}, J_{1^{\prime}, 2^{\prime}}=9.2 \mathrm{~Hz}, \mathrm{H}-2^{\prime}\right), 5.63\left(1 \mathrm{H}, \mathrm{dd}, J_{3^{\prime}, 4^{\prime}}=8.8 \mathrm{~Hz}, J_{2^{\prime}, 3^{\prime}}=1.6 \mathrm{~Hz}, \mathrm{H}-3^{\prime}\right), 5.06(1 \mathrm{H}$, m, H-4'), $4.24\left(1 \mathrm{H}, \mathrm{dd}, J_{4^{\prime}, 5^{\prime}}=2.8 \mathrm{~Hz}, J_{5^{\prime}, 5^{\prime \prime}}=12.1 \mathrm{~Hz}, \mathrm{H}-5^{\prime}\right), 4.11\left(1 \mathrm{H}, \mathrm{dd}, J_{4^{\prime}, 5^{\prime \prime}}=4.7 \mathrm{~Hz}, J_{5^{\prime}, 5^{\prime \prime}}\right.$ $\left.=12.2 \mathrm{~Hz}, \mathrm{H}-5^{\prime \prime}\right), 2.26,2.20,2.19,2.01,1.99,1.80\left(\right.$ each $\left.3 \mathrm{H}, 6 \mathrm{~s}, 6 \mathrm{CH}_{3}\right) ;{ }^{13} \mathrm{C} \mathrm{NMR}\left(\mathrm{CDCl}_{3}\right): \delta$ 170.3-168.1 $\left(\mathrm{OCOCH}_{3}\right), 152.3$ (C-2), 136.1 (C-1), 128.7 (C-4), 128.3 (C-5), 127.7 (C-3), 118.4 (C-6), 70.0 (C-1'), 68.3, 67.4, 66.7 (C-2', C-3', C-4'), 62.0 (C-5'), 20.8, 20.4, 20.4, $20.0\left(\mathrm{CH}_{3}\right)$.

3-Hydroxy-2-nitrobenzaldehyde (16f). ${ }^{30}$ To a solution of 3-(1',2',3', 4', 5' -penta- $O$-acetyl-Dgalacto-pentitol-1'-yl)-2-nitrophenol (16a, $0.50 \mathrm{~g}, 1.00 \mathrm{mmol})$ in $\mathrm{MeOH}(90 \%, 15 \mathrm{~mL})$ was added $\mathrm{K}_{2} \mathrm{CO}_{3}(0.50 \mathrm{~g}, 3.62 \mathrm{mmol})$. After stirring at room temperature for $24 \mathrm{~h}$, TLC (Et $2 \mathrm{O}-$ petroleum ether, 2:1) showed complete consumption of $16 \mathbf{a}$ with one product at $R_{f} 0.20$ present. Neutralization with Amberlite IR-120 $\left(\mathrm{H}^{+}\right)$and evaporation of the solvent gave a colorless oil, which was dissolved in $\mathrm{MeOH}(50 \%, 20 \mathrm{~mL})$. The solution was stirred with $\mathrm{NaIO}_{4}(1.00 \mathrm{~g}, 4.67$ $\mathrm{mmol})$ at room temperature for $15 \mathrm{~min}$. Then, the mixture was extracted with $\mathrm{CH}_{2} \mathrm{Cl}_{2}(3 \times 20$ $\mathrm{mL})$, the combined extracts were washed with water $(3 \times 20 \mathrm{~mL})$, dried and evaporated to yield an oil, which was purified by column chromatography ( $\mathrm{Et}_{2} \mathrm{O}$-petroleum ether, 1.5:1). Crystallization from petroleum ether afforded a solid identified as 3-hydroxy-2-nitrobenzaldehyde $(0.090 \mathrm{~g}, 54 \%)$; mp $150-152{ }^{\circ} \mathrm{C}$ (lit. $\left.{ }^{30} \mathrm{mp} 150-151{ }^{\circ} \mathrm{C}\right) ; R_{f} 0.29\left(\mathrm{Et}_{2} \mathrm{O} /\right.$ petroleum ether, 2:1); IR (KBr) $\tilde{v}_{\max }\left(\mathrm{cm}^{-1}\right): 3600(\mathrm{OH}), 3030\left(\mathrm{H}-\mathrm{C}_{\mathrm{ar}}\right), 2815(\mathrm{C}-\mathrm{H}$ aldehyde $) 1715(\mathrm{C}=\mathrm{O}), 1530$, $1330\left(\mathrm{NO}_{2}\right) ;{ }^{1} \mathrm{H}$ NMR $\left(\mathrm{CDCl}_{3}\right): \delta 10.19(1 \mathrm{H}, \mathrm{s}, \mathrm{CHO}), 9.75\left(1 \mathrm{H}, \mathrm{s}, \mathrm{D}_{2} \mathrm{O}\right.$ exchangeable $\left.\mathrm{OH}\right), 7.71$ $\left(1 \mathrm{H}, \mathrm{t}, J_{5,6}=7.5 \mathrm{~Hz}, \mathrm{H}-5\right), 7.62\left(1 \mathrm{H}, \mathrm{d}, J_{5,6}=7.5 \mathrm{~Hz}, \mathrm{H}-6\right), 7.25\left(1 \mathrm{H}, \mathrm{d}, J_{4,5}=7.5 \mathrm{~Hz}, \mathrm{H}-4\right) ;{ }^{13} \mathrm{C}$ NMR (CDCl $)$ : $\delta 193.4$ (CHO), 155.3 (C-3), 140.0 (C-2), 136.2 (C-5), 132.3 (C-1), 124.3, 122,5 (C-4, C-6).

By this procedure, 3-hydroxy-2-nitrobenzaldehyde (16f; 61\%) was also obtained from $\mathbf{1 6 b .}$

\section{Acknowledgements}

We thank Ministerio de Educación y Ciencia, Dirección General de Investigación through grants BQU2003-05946 and CTQ2005-07676/BQU. We also thank the Junta de Extremadura for a fellowship to N. Araújo. 


\section{References}

1. Rebstock, M. C.; Crooks, Jr. H. M.; Controulis, J.; Bartz, Q. R. J. Am. Chem. Soc. 1949, 71, 2458.

2. Beard, N. S.; Armentrout, S. A.; Weisberger, A. S. Pharmacol. Rev. 1969, 21, 213.

3. Lampis, G.; Deidda, D.; Maullu, C.; Petruzzelli, S.; Pompei, R. J. Antibiot. 1996, 49, 263.

4. Chen, J.; Sambaiah, T.; Illarionov, B.; Fischer, M.; Bacher, A.; Cushman, M. J. Org. Chem. 2004, 69, 6996.

5. Alahiane, A.; Rochdi, A.; Taourirte, M.; Redwane, N.; Sebti, S.; Lazrek, H. B. Tetrahedron Lett. 2001, 42, 3579.

6. Shaban, M. A. E.; Nasr, A. Z.; Morgaan, A. E. A. Pharmazie 2000, 55, 87.

7. Bari, A.; Milicevic, S.; Feist, H.; Michalik, D.; Michalik, M.; Peseke, K. Synthesis 2005, 2758.

8. Nasr, A. Z. Nucleosides, Nucleotides and Nucleic Acids 2004, 23, 1825.

9. Hanessian, S.; Haskell, T. H. In The Carbohydrates: Chemistry and Biochemistry, Vol II A. Pigman, W.; Horton, D., Eds., Academic Press, New York, 1970.

10. Hanessian, S.; Pernet, A. G. Adv. Carbohydr. Chem. Biochem. 1976, 33, 111.

11. Suhadolnik, R. J. Nucleoside Antibiotics, Wiley-Interscience, New York, 1970.

12. White, H. R. J. Am. Chem. Soc. 1986, 108, 6434.

13. Araújo, N.; Gil, M. V.; Román, E.; Serrano, J. A. Synthesis 2006, 2503.

14. Areces, P.; Jiménez, J. L.; Pozo, M. C.; Román, E.; Serrano, J. A. J. Chem. Soc., Perkin Trans. 1 2001, 754.

15. Serrano, J. A.; Cáceres, L. E.; Román, E. J. Chem. Soc., Perkin Trans. 1 1995, 1863, and references cited therein.

16. Sowden, J. C.; Strobach, D. R. J. Am. Chem. Soc. 1960, 82, 954.

17. Sowden, J. C.; Schaffer, R. J. Am. Chem. Soc. 1950, 73, 4662.

18. Brion, F. Tetrahedron Lett. 1982, 23, 5299.

19. Xing, Y. D.; Haung, N. Z. J. Org. Chem. 1982, 47, 140.

20. Maeba, I.; Usami F.; Furukawa, H. J. Org. Chem. 1984, 49, 1534.

21. (a) The Aldrich Library of Infrared Spectra, Ed. III, Aldrich Chemical Company, Wisconsin, 1981, p. 912. (b) The Aldrich Library of NMR Spectra, Vol. 2, Ed. II, Aldrich Chemical Company, Wisconsin, 1983, p. 105. (c) Tsang, S. M.; Wood, E. H.; Johnson, J. R. Organic Synthesis Coll., Vol. 3, John Wiley \& Sons, New York, 1995, p. 641.

22. Cowan, J. A. Tetrahedron Lett. 1986, 27, 1205.

23. Jackman, L. M.; Sternhell, S. Applications of Nuclear Magnetic Resonance Spectroscopy in Organic Chemistry, 2nd ed., Pergamon Press, New York, 1969, p. 203.

24. Bobek, M.; Farkaš, J.; Sorm, F. Collect. Czech. Chem. Commun. 1969, 34, 1673.

25. (a) Bock, K.; Pedersen, C. Adv. Carbohydr. Chem. Biochem. 1983, 41, 27. (b) Bueno Martínez, M.; Román Galán, E.; Galbis Pérez, J. A. Nucleosides and Nucleotides 1988, 7, 227. 
26. Barker, R.; Hudson, B. G. J. Org. Chem. 1967, 32, 3650.

27. (a) Izumi, K. Carbohydr. Res. 1973, 27, 278. (b) Duclos, A.; Fayet, C.; Gelas, J. Synthesis 1994, 1087. (c) Hama, Y.; Nakanawa, H.; Sumi, T.; Xia, X.; Yamaguchi, K. Carbohydr. Res. 1999, 318, 154.

28. Román-Galán, E.; Galbis-Pérez, J. A.; Arévalo-Arévalo, M. A. Carbohydr. Res. 1983, 116, 255.

29. Butterworth, R. F.; Hanessian, S. Synthesis 1971, 70.

30. (a) Hodgson, T.; Smith, H. J. Chem. Soc. 1937, 77. (b) Nozoe, T.; Mukai, T.; Sakai, K. Tetrahedron Lett. 1965, 6, 1041.

31. Harwood, L. M. Aldrichim. Acta 1985, 18, 25. 